\title{
Neuroendocrine neoplasia and bone (Review)
}

\author{
ADINA GHEMIGIAN ${ }^{1,2}$, MARA CARSOTE ${ }^{1,2}$, FLORICA SANDRU ${ }^{3,4}$, RAZVAN-COSMIN PETCA $^{5,6}$, \\ ANA-MARIA OPROIU ${ }^{7,8}$, AIDA PETCA ${ }^{9,10}$ and ANA VALEA ${ }^{11,12}$ \\ ${ }^{1}$ Department of Endocrinology, 'Carol Davila' University of Medicine and Pharmacy, 050474 Bucharest; \\ ${ }^{2}$ Department of Endocrinology, 'C. I. Parhon' National Institute of Endocrinology, 011863 Bucharest; \\ ${ }^{3}$ Department of Dermatology, 'Carol Davila' University of Medicine and Pharmacy, 050474 Bucharest; \\ ${ }^{4}$ Department of Dermatology, 'Elias' Emergency University Hospital, 011461 Bucharest; ${ }^{5}$ Department of Urology, \\ 'Carol Davila' University of Medicine and Pharmacy, 050474 Bucharest; ${ }^{6}$ Department of Urology, \\ 'Prof. Dr. Theodor Burghele' Clinical Hospital, 061344 Bucharest; ${ }^{7}$ Department of Plastic and Reconstructive Surgery, \\ 'Carol Davila' University of Medicine and Pharmacy, 050474 Bucharest; ${ }^{8}$ Department of Plastic and Reconstructive Surgery, \\ University Emergency Hospital, 050098 Bucharest; ${ }^{9}$ Department of Obstetrics and Gynecology, 'Carol Davila' University of \\ Medicine and Pharmacy, 050474 Bucharest; ${ }^{10}$ Department of Obstetrics and Gynecology, University Emergency Hospital, \\ 050098 Bucharest; ${ }^{11}$ Department of Endocrinology, 'I. Hatieganu' University of Medicine and Pharmacy, \\ 400012 Cluj-Napoca; ${ }^{12}$ Department of Endocrinology, Clinical County Hospital, 400000 Cluj-Napoca, Romania
}

Received June 29, 2021; Accepted July 29, 2021

DOI: $10.3892 / \mathrm{etm} .2021 .10653$

\begin{abstract}
This is a narrative review focusing on neuroendocrine neoplasia (NEN) and bone status, in terms of metastases and osteoporosis/fractures. One fifth of NEN have skeletal

Correspondence to: Dr Florica Sandru, Department of Dermatology, 'Elias' Emergency University Hospital, 17 Mărăști Boulevard, 011461 Bucharest, Romania

E-mail: florysandru@yahoo.com
\end{abstract}

Abbreviations: ACTH, adrenocorticotropic hormone; BMD, bone mineral density; CT, computed tomography; CXCR4, C-X-C chemokine receptor 4; DXA, dual-energy X-ray absorptiometry; EMT, epithelial-mesenchymal rransition; ${ }^{18} \mathrm{~F}-\mathrm{FDG}$, 18-F-fluoro-deoxy-glucose; FRAX, fracture risk assessment tool; PHEO, pheocromocytoma; Ga, gallium; GEP-NEN, gastroenteropancreatic neuroendocrine neoplasia; 5-HIIA, 5-hydroxyindolacetic acid; 25(OH)D, 25-hydroxyvitamin D; $\mathrm{I}^{131}$-MIBG, radioiodine 131-metaiodobenzylguanidine; IGF1, insulin-like growth factor 1; IOF, International Osteoporosis Foundation; MEN, multiple endocrine neoplasia; MiNENs, mixed non-neuroendocrine and neuroendocrine differentiation; NEN, neuroendocrine neoplasia; NEC, neuroendocrine carcinoma; NET, neuroendocrine tumor; PET/CT, positron emission tomography/computed tomography; PTH, parathormone; PTHrP, parathormone-related protein; PG, paraganglioma; PHP, primary hyperparathyroidism; SRS, somatostatin receptor scintigraphy; SSRI, selective serotonin reuptake inhibitors; SDHB, succinate dehydrogenase; TBS, Trabecular Bone Score; WHO, World Health Organization

Key words: neuroendocrine neoplasia, carcinoid, fractures, osteoporosis, bone, dual-energy X-ray absorptiometry, serotonin, ectopic Cushing syndrome, pheocromocytoma dissemination, this affinity being regulated by intrinsic tumor factors such as the C-X-C chemokine receptor 4 (CXCR4). Bone colonization impairs the patient quality of life, representing a surrogate of reduced survival. Patients with NEN without bone metastases may exhibit low bone mineral density, perhaps carcinoid-related osteoporosis, yet not a standardized cause of osteoporosis. Case-finding strategies to address bone health in NEN with a good prognosis are lacking. Contributors to fractures in NEN subjects may include: menopausal status and advanced age, different drugs, induced hypogonadism, malnutrition, malabsorption (due to intestinal resection, carcinoid syndrome), hypovitaminosis D, impaired glucose profile (due to excessive hormones such as glucagon, somatostatinoma or use of somatostatin analogues), various corticoid regimes, and high risk of fall due to sarcopenia. Pheocromocytoma/paraganglioma involve bone through malignant forms (bone is an elective site) and potential secondary osteoporosis due to excessive hormonal content and increased sympathetic activity which is a key player of bone microarchitecture/quality as reflected by low Trabecular Bone Score. Glucocorticoid osteoporosis is related to NEN-associated ectopic Cushing syndrome. Currently, there are a lack of studies to emphasis that excessive gut-derivate serotonin in NENs with carcinoid syndrome is a specific activator of bone loss thus a contributor to carcinoid-related osteoporosis.

\section{Contents}

1. Introduction

2. Aim of the review

3. Neuroendocrine neoplasia: A dynamic yet heterogeneous domain

4. Osteoporosis and osteoporotic fractures: More than an economic burden 

5. Skeleton-related events in NENs with metastatic potential
6. Carcinoid-related osteoporosis
7. Pheocromocytoma/paraganglioma and skeleton
8. NEN-associated ectopic Cushing syndrome
9. Further considerations
10. Conclusion

\section{Introduction}

Neuroendocrine neoplasia (NEN) represents a challenging field, continuing the traditional concept of 'karzinoide' introduced by Oberndorfer in 1907, that was followed by the concept of neuroendocrine tumors (NETs) and then NEN according to different WHO (World Health Organization) classifications (mainly 2010, 2017, 2019) that currently takes into consideration the Ki67 and mitotic index for grading (1-3). Basically, according to the 2019 WHO classification, NETs are included in the group of NENs of gastrointestinal tract origin in addition to neuroendocrine carcinoma (NEC), also defining mixed non-neuroendocrine and neuroendocrine differentiation tumors (MiNENs) (4).

Any organ has the potential to host a tumor of neuronal embryologic origin with endocrine features, more or less associated with clear clinical and biochemical expression such as classical carcinoid syndrome. Yet, actually, NENs, most of the time portend lung and gastro-entero-pancreatic (GEP) NENs (5). The heterogeneity of the domain and new discoveries continuously require novel nomenclature, while a wide area of different practitioners are dedicated to the field of NENs (4-6). Impressive progress has been registered currently, thus an improved prognosis and a longer survival rate are reported in regards to many subtypes $(7,8)$. The tumors underline a large spectrum of malignant potential, although the traditional benign-malign cutoffs are not really applicable in this area; however Ki67 labeling based on immunohistochemistry reports, overall NEN grading, remains the key indicator of aggressive behavior in association with the type of originating tissue, to local and distance spreading, and to the therapeutic approach that is feasible for a patient in point $(7,8)$.

\section{Aim of the review}

This is a narrative review of literature focusing on NEN and bone status, in terms of both skeletal metastases but also fractures and the associated diagnosis of osteoporosis. The main (but not exclusive) research tool was PubMed. The key words were 'neuroendocrine neoplasia', but also, due to changes of terminology over the years, 'neuroendocrine tumors' and 'carcinoid tumors' in addition to various combinations of 'bone', 'skeletal', 'metastases', 'fractures', 'osteoporosis'. In addition, a collateral research included 'ectopic Cushing syndrome', 'pheocromocytoma', and 'paraganglioma' and bone field. The objective was to provide practical points for different practitioners in this complex, trans-disciplinary topic. The cited articles were mostly published between 2010 and 2020. A number of 119 references were introduced. The data were organized in micro-chapters reflecting the current status of the NEN field, as well as osteoporosis; then the relationship between NEN-associated bone metastases, respective carcinoid-related osteoporosis; and finally two topics of overlap between NEN and skeleton aspects: pheocromocytoma/paraganglioma (PHEO/PG) and ectopic Cushing syndrome.

\section{Neuroendocrine neoplasia: A dynamic yet heterogeneous domain}

Reports of NEN incidence vary, but generally propose an increasing trend due to endocrine disruptors, environmental triggers and advances in detection and screening protocols (9-11). The low incidence in the global population has increased to 6 times in the US during the last 30 years (9). A study on 6,179 NETs showed that the incidence between 1995 and 2012 went from 3.1 to 7.1/100,000 cases (or 3.96 to 6.61, depending on database); the most frequent age interval was between 50 and 64 years; most often NET origins are 30.6\% for lung, respective $16.82 \%$ for the small intestine (9).

Another database between 1995 and 2014 included 85,133 NETs and it showed that the G1 NET median overall survival was 233 months with a 5-year NEN-specific survival rate of 97.6\%; median survival decreased with age (poor prognosis was registered especially in individuals over 70 years); $34 \%$ of persons over 70 years had metastases at diagnosis and $40.8 \%$ of these patients presented with G3 NET (10). Data from the Swedish Cancer Registry revealed the following related to the metastatic potential of 7,334 NENs. Liver was the most frequent site (representing $82 \%$ of all the patients with NEN-related metastases); $66 \%$ of patients with lung metastases had synchronous hepatic spreading; small intestine followed by pancreatic-hepatic-biliary tract were the most common histological types that were associated with metastases while the lowest metastatic potential was for appendix and rectal NENs; males had more frequent metastases than females; the most severe prognosis was in subjects with metastases from unknown primary NEN; and the metastatic behavior mostly depended on the primary tumor site (11).

As this study, and also another study on 302 NENs, brain metastases were most frequently associated with lung NEN, and most probably at that point, liver and other sites such as the lymph nodes and bone metastases were synchronously positive $(11,12)$. Bone metastases were described in one fifth of the patients diagnosed with NEN (13). The skeleton affinity was regulated by intrinsic factors of epithelial-mesenchymal transition (EMT) such as $\mathrm{C}-\mathrm{X}-\mathrm{C}$ chemokine receptor 4 (CXCR4) expression, in collaboration with other cofactors such as CXCL12 (13). To date, the complex biomolecular mechanisms of skeleton spreading are less understood, yet the point is that bone colonization impairs the quality of life of the patient and it serves as a surrogate for a reduced survival rate (14).

\section{Osteoporosis and osteoporotic fractures: More than an economic burden}

Whether there is a relationship between NENs without bone metastases and associated low bone mass (a potential new cause of secondary osteoporosis) and primary osteoporosis (menopausal or age-related) is still difficult to establish based on the epidemiological data we have to date. Contrary to the 
low NEN incidence in the global population (despite the recent massive increasing trend), primary osteoporosis and associated low-trauma (osteoporotic) fractures have a high prevalence in the general population, especially in the aging population, in postmenopausal women and seniors (which also represent the more exposed population for the diagnosis of NENs) (15-17).

According to data from the International Osteoporosis Foundation (IOF) site, the number of people at high fracture risk from 2010 will double in 2040; 1 in 3 females and 1 in 5 males over 50 years will suffer an osteoporotic fracture (18). The economic burden related to low-trauma fractures is reflected in associated costs of $\$ 17.9$ up to $£ 4$ billion/year in the UK and the US due to the fact that currently osteoporosis is undertreated (19). These days, less than one fifth of osteoporotic patients receive medication for this diagnosis, and the current rate is not increasing due to multiple causes such as the presence of other co-morbidities (especially age-dependent) that also require medication where osteoporosis is not considered a priority; the fear of side effects of anti-osteoporotic drugs; and difficulties of anti-osteoporosis medication reimbursement protocols depending on countries (20).

Secondary prevention (treatment of osteoporosis after a first osteoporotic fracture) also is suboptimal (21). For instance, a picture of how the real world medical approach has emerged, is reflected by a study in 2019 on 2,933 patients over 50 years of age with a recent incident fragility vertebral fracture (between 2008 and 2014). This study showed that $93 \%$ of these patients were naïve of anti-osteoporosis medication during the prior 2 years and the following year after the diagnosis of the fracture (21). Only $7 \%$ of patients immediately started medication after fracture and another $5 \%$ of subjects were offered drugs during the first year, while $73 \%$ of them were never treated (21). A total of $38 \%$ of these patients had a second fracture within the following 24 months (21). Screening programs may be helpful. For example, a controlled study from 2020 included UK people aged between 70 and 85 years showing that the screening of 1,000 subjects saved 29 fractures (hip/non-hip ratio of 9/20) during the following 14 years (remaining life time) with a significant improvement in the associated costs (22). A multitude of factors are contributors to damaged bone mass, especially after the age of 50 years. Based on the epidemiological data that we have to date, NENs are not yet an input in this playlist but convincing evidence is increasing.

\section{Skeleton-related events in NENs with metastatic potential}

NENs may affect the skeleton status through different mechanisms $(23,24)$. On the one hand, there are well-established malignancy-related aspects which include: bone metastases potentially associated with pathological fractures (such as vertebral fractures or even atypical femoral fractures), vertebral/cord compression due to vertebral fractures, and paraneoplasic hypercalcemia $(23,24)$. The natural history of such incidental events largely varies due to the multiple subtypes of NENs; thus, a different behavior is expected, but the most important aspect in secondary dissemination is actually the tumor aggressive pattern rather than a particular histological type $(25,26)$.
Generally, the presence of bone metastases in patients with NENs is regarded as a poor prognostic indicator and the local behavior typically does not differ in regards to the bone metastases of other non-NEN malignancies (23). If liver and lung metastases are already identified, then skeleton and brain metastases should be checked (27). A potential explanation of the fact that some NENs have a high bone affinity is the overexpression of CXCR4 at the level of the tumor and its serial identification through immunohistochemistry after surgical removal or after biopsy may become a predictive factor of skeleton dissemination (26).

A multi-institution, 5-year study analyzed the time-to-event parameters in 691 patients with NENs and 82 of them (representing $12 \%$ ) had bone metastases (28). A total of $55 \%$ of the patients with bone metastases were males; median age at diagnosis was 49 years; among NENs: $25 \%$ were malignant pheocromocytoma and paragangliomas, $20 \%$ were G3 NENs, while $8 \%$ had pancreatic origin (28). The complications of metastases were the following: local symptoms, mostly pain $(60 \%)$, cord compression $(10 \%)$, secondary fractures $(9 \%)$, and hypercalcemia (4\%) (28). Their overall management included: local radiation therapy $(50 \%)$, bisphosphonates $(45 \%)$, bone/spine surgery (18\%), denosumab (5\%); as for malignant PHEO/PG additional ${ }^{131}$-MIBG (radioiodine 131-metaiodobenzylguanidine) therapy was introduced (accounting for $11 \%$ of all NEN patients with bone metastases) (28).

A prior review published by Hori et al showed that the average age of patients with carcinoid tumor-related skeleton metastases was 54.9 years, with male predominance $(66 \%)$; most frequent bone site was the vertebra; the survival rate was lower in osteolytic type compared to osteosclerotic metastases (29). Another study published in 2020 on 2,005 NEN patients showed a prevalence of $7 \%$ for bone metastases, representing an independent factor of poor survival based on a hazard ratio (HR) of 1.412 (95\% CI: 0.965-2.065) (30). Another study from 2019 included 3,909 pancreatic NENs and it showed that, while the most metastatic organ was the liver, the median survival time when bone metastases were present was 56 months and in this situation the 5-year overall survival was $42.7 \%$ (31).

An imaging based study from 2020 analyzed bone metastases detected with ${ }^{18} \mathrm{~F}$-DOPA PET in 155 metastatic midgut NEN patients (admitted between 2011 and 2018) and it showed that: $29.7 \%$ of NENs had skeleton colonization; bone dissemination was independently associated with carcinoid syndrome; the majority $(78 \%)$ were of osteoblastic subtype; less than $9 \%$ were symptomatic; they represented an independent prognostic factor (32). The concept of 'oligo-bone metastases' was introduced for lesions associated with less than 5 criteria at ${ }^{18} \mathrm{~F}$-DOPA PET (vs. 'poly-bone metastases') and they were not associated with a poor prognosis (opposite to 'poly' subtype) (32). Overall, NENs may embrace a metastatic profile, nevertheless including the bone, thus a level of awareness is essential.

\section{Carcinoid-related osteoporosis}

On the other hand, non-metastatic skeleton damage may include the traditional diagnosis of osteoporosis/osteopenia according to central dual-energy X-ray absorptiometry 
(DXA) and potentially complicated with (low-trauma or even spontaneous) osteoporotic fractures, in tumors such as lung and GEP-NEN, due to multiple hormonal, mechanical, nutritional, and metabolic factors (33). We propose the term carcinoid-related osteoporosis, even though the actual traditional terminology of 'carcinoid' tumor has been replaced by NEN/NET; yet, we consider that this expression clarifies a new clinical entity, different from neuroendocrine mechanisms underlying bone status under normal and abnormal conditions $(34,35)$. The pathogeny and epidemiologic data are still a matter to discuss, while a correlation with NEN status itself or with neuroendocrine marker levels is ongoing $(34,35)$.

A few studies have shown that in NENs, serum serotonin, not its urinary metabolite hydroxyindolacetic acid (5-HIIA), is inversely correlated with hip BMD (34). But the skeleton influence consists in a cocktail of factors produced by the tumor, including non-serotonin growth factors or hormones with bone tropism (35). Up to this moment, carcinoid-related osteoporosis is not a standardized cause of secondary osteoporosis, but there is an increasing amount of medical evidence that points out this well-deserved specification. Case-finding strategies are still lacking in order to address the bone health in these cases, especially in those with a good prognosis, and in the absence of bone metastases. The importance of approaching the bone issues in addition to medical and surgical management in NEN subjects is related to increased quality of life knowing that adequate protocol of multimodal investigations as well as modern multiple-level NEN therapies have massively improved the overall survival during the last decade $(36,37)$.

The tools of bone and fracture risk assessment, as seen in non-NEN cases, are mostly similar: the evaluation of phosphorus-calcium metabolism: serum calcium and phosphate levels, mineral hormones (parathormone (PTH) and 25-hydroxyvitamin D [25(OH)D]], bone turnover markers [of formation (alkaline phosphatase, osteocalcin, P1NP] and of resorption (CrossLaps) $(38,39)$. Also, traditional fracture risk assessment includes central DXA (especially in menopausal women and men over 50 years old), while the Fracture risk assessment tool (FRAX) model does not include as distinct input the diagnosis of NEN $(40,41)$. As regard to the Trabecular Bone Score (TBA), data are also missing, except for an extrapolation for a diabetic subpopulation (especially in post-menopausal females) of NEN subjects due to impaired glucose profile via the secretion of hyperglycemic hormones such as glucagon, also for syndromic circumstances that are associated with primary hyperparathyroidism or for Cushing syndrome as seen in paraneoplastic type accompanying some NENs $(42,43)$. In some NEN multi-leveled regimes, glucocorticoids are used and thus TBS-related changes are expected (44).

The identification of prevalent fractures starts from a simple screening plane X-ray of the spine (for instance, profile thorax-lumbar X-ray) up to computed tomography (CT) or magnetic resonance imaging (MRI) (that are usually applied in the non-NEN general population in selected cases) or in addition to detailed investigations for NENs such as whole body bone scintigraphy, somatostatin receptor scintigraphy (SRS) or positron emission tomography/computed tomography (PET-CT) which may also serve for differentiation of a metastasis-related fracture from an osteoporotic fracture $(45,46)$.

In terms of skeleton health, patients with NENs may suffer from a multitude of factors, not only from direct malignant dissemination to the bone $(47,48)$. For instance, secondary diabetes mellitus due to pancreatic and duodenal tumors such as somatostatinomas (with or without underlying neurofibromatosis type 1), or pheocromocytomas or due to paraneoplastic hypercortisolemia may cause a deterioration of bone quality like that reflected by TBS in addition to abnormal turnover that increase fracture risk (49-51). In addition, some worsening of the glucose profile is expected after high doses of somatostatin analogues, a powerful medication for NEN control of secretion and growth, as seen (to a lesser extent though) in acromegaly $(52,53)$.

Weight loss is another mechanism of bone mass decrease, being caused by long standing disease-related anorexia, different oncological drugs such as chemotherapy, or intestinal/gastric resection because of tumor location (54). Weight loss may be associated with other nutritional deficiency and anemia of different mechanisms (55).

Low body mass index is associated with low BMD and high fracture risk, as also reflected by the algorithm FRAX, as generally applicable in individuals over 40 years of age $(56,57)$. Weight loss, advanced age, some drugs (such as checkpoint inhibitors), uncontrolled carcinoid syndrome (clinically manifested with diarrhea) and severe illness may aggravate a process of sarcopenia (deficiency of skeletal muscle mass and strength) that is a supplementary contributor to the risk of fall and an independent indicator of poor prognosis (58). Some chemotherapy regimens and radiotherapy are associated with dexamethasone therapy or similar glucocorticoid products, thus a component of glucocorticoid osteoporosis may be overlapped under these particular circumstances (59). In NENs of prostatic or ovarian origin, the co-presence of hormonal-dependent adenocarcinoma may require induction of hypogonadism, either chemically or surgically, causing low BMD (60). Hypovitaminosis D, a particular risk factor of poor bone status, is related to malnutrition, surgery of the intestinal area, malabsorption, liver metastasis, low sun exposure, and different concurrent medications (61). Of course, NEN itself may produce hormones that have a negative impact on the skeleton such as serotonin or parathormone-related protein (PTHrP) $(62,63)$.

\section{Pheocromocytoma/paraganglioma and skeleton}

PHEO, respective PG, represent tumors with adrenal, respective extra-adrenal enterochromaphin cells which produce catecholamine (norepinephrine and epinephrine) and others amines like dopamine. These tumors are typically candidates to surgery in order to be cured, associating a high cardiovascular risk of complications before, during and after surgery $(64,65)$. Neoplasia has a low incidence in the global population (2-8/1 million) but they represent up to 6 cases out of 1,000 hypertensive persons (66). However, in otherwise asymptomatic patients, they may actually be accidentally detected as an incidentaloma in $10-50 \%$ of cases, depending on the definition of incidentaloma (67). Metastases define the malignant potential, and not necessarily the post-operative 
histological report $(66,68)$. The percent of distance spreading varies between 2 and $60 \%$, most frequently 3-10\% depending on the study, usually PG having a higher potential than PHEO (66-69).

Bone is the most frequent site of metastases, involving different areas such as the vertebras to unusual locations such as the mandibular or orbital $(70,71)$. If succinate dehydrogenase (SDHB) is positive, the metastatic rate is higher than in sporadic cases; other sites of malignant colonization are the lung and liver with a more severe prognosis (69). Skeletal metastases may be positive at first admission or at a more distant time (even after 2 decades since diagnosis) (72). Some cases of metastatic spine paraganglioma inducing cord compression have been reported (73). Skeletal-related events may also include hypercalcemia due to bone metastases or PTHrP tumor production, distinct to the synchronous presence of primary hyperparathyroidism (PHP) in cases with type 2A multiple endocrine neoplasia (MEN) syndrome $(74,75)$. Removal of the primary tumor improves the prognosis, while prevention of metastases is less feasible, but prompt extended interventional radiology methods may help (76).

In non-malignant PHEO/PG, several studies have shown a higher prevalence of osteoporosis/osteoporotic fracture compared with the general population, related to the tumor hormonal over-secretion with the bone targeted and to increased sympathetic activity which is a key player of bone microarchitecture/quality (TBS assay may reflect this particular aspect) (77-79). Thus an improvement in bone status after tumor removal has been observed (77-79).

A retrospective, single-center study in 2020 showed in 49 patients with PHEO vs. 61 subjects with non-secretor adrenal tumors that prevalence of vertebral fractures was higher (43 vs. 16\%) after adjustment for age and sex, with a $3.8 \%$ increase in lumbar BMD after tumor removal (79). A controlled study in adrenal tumors (incidentaloma/PHEO ratio of 266/29) showed a $2.9 \%$ lumbar TBS reduction in subjects with catecholamine-producing tumors who also had a statistically significant reduced lumbar BMD and an increased collagen-derivate bone resorption marker (78).

Another study from 2021 published by Q-AND-A study group also showed a lower TBS in the PHEO/PG group vs. non-functional adrenal tumors; TBS being statistically significant lower in subjects with vertebral fractures vs. those without vertebral osteoporotic fractures; TBS was independent to BMD decrease; TBS was inversely correlated with hormone excess and tumor size (80). The same study group showed that PHEO patients had a higher rate of abdominal aortic calcifications when associated with vertebral fractures, thus proving the co-presence of osteoporosis and atherosclerosis in PHEO (81). In primary menopausal osteoporosis, a link between metabolism disturbances such as hyperglycemia or hyperlipidemia and low BMD and/or low TBS has already been established $(82,83)$. Specific protocols of BMD/TBS screening in $\mathrm{PHEP} / \mathrm{PG}$ patients, especially over the age of 50 years or menopausal woman are still deficient.

\section{NEN-associated ectopic Cushing syndrome}

Endogenous Cushing syndrome, of either adrenal, pituitary or cancer-related cause (including NEN), is a combination of obesity, diabetes mellitus, high cardiovascular risk, hypokalemia, coagulation disturbances, hirsutism, male hypogonadism, high frequency of infections, secondary glucocorticoid osteoporosis and associated low-trauma fractures such as vertebral fractures, and also aseptic necrosis of different regions as the hip or shoulders $(84,85)$. The rapid evolution of the symptoms, and a phenotype likely associated with imbalances in serum electrolytes, hyperpigmentation, weight loss, and proximal myopathy are more suggestive of paraneoplastic Cushing syndrome (86). The neoplasia mass itself and potential local effects of the originating tumor may be identified or the underlying cause remains occult, thus multiple investigative protocols are necessary to locate it, including functional imaging (87). In addition to identification of the primary source of non-pituitary adrenocorticotropic hormone (ACTH), tests are first necessary to confirm that it is an ectopic Cushing syndrome (such as steroid metabolomic assays) (88). As management, ideally the removal of NEN is recommended to control hypercortisolemia, but in many cases it is not feasible thus steroidogenesis inhibitors, mitotane or bilateral adrenalectomy are indicated to control the large area of complications due to persistent exposure to hypercorticism (89).

Fragility fractures occur in Cushing syndrome due to anomalies of bone turnover as reflected by bone turnover markers (increased bone resorption, reduced bone formation), anomalies of growth hormone [insulin-like growth factor (IGF1)], of sclerostin, and potentially due to the effects of diabetes mellitus on bone, and even weight changes in association or not with hypovitaminosis D $(90,91)$.

A cohort study between 2000 and 2019 included 130 subjects with ectopic Cushing syndrome (female/male ratio of 93/37) and it showed that the prevalence of osteoporosis was 25 vs. $48 \%$ (females vs. males), while the prevalence of vertebral fractures was 5 vs. $16 \%$, suggesting the fact that actually males are more affected than women when it comes to bone status (92). Moreover, the fracture risk was dependent to some extend on low BMD according to central DXA and/or decreased values of TBS; but the main contributor to fracture risk was found to be the level of hypercortisolemia and the time of exposure, not necessary the pathogenic type of Cushing syndrome or the source of ectopic ACTH over-secretion (meaning that it seemed less important that there was an underling NEN in association with ectopic ACTH production in terms of glucocorticoid osteoporosis) (93).

The European Registry on Cushing's syndrome (ERCUSYN) collected the data of 481 patients with Cushing syndrome, from 36 centers, respective 23 countries, between 2000 and 2008, and 5\% of them had ectopic ACTH excess (94). Overall, the prior mentioned observation was consistent; male vs. women had a statistically significant higher frequency of osteoporosis based on lumbar BMD, and more often were diagnosed with vertebral fractures (52 vs. 18\%), and also rib fractures (34 vs. 23\%) (94).

Among NENs, patients with bronchial carcinoid were more frequently reported to develop ectopic Cushing syndrome, potentially complicated with osteoporotic fractures especially at the vertebral site, even complicated with vertebral compression that required surgical procedures such as vertebroplasty (95). Another scenario included the synchronous 
presence of NEN-related paraneoplastic Cushing syndrome with potential skeleton impairment and an NEN with a gastro-intestinal origin, or even of thymus origin or medullary thyroid cancer, with bone metastases $(96,97)$. Generally, when bone metastases are positive, NEN liver spreading is already present, thus there is also a higher risk of an associated clinically manifested carcinoid syndrome due to interferences in NEN-released serotonin hepatic metabolism $(98,99)$. The differentiation of the etiology of the fractures (osteoporotic or due to malignancy spreading) may be assessed by using advanced technology tools such as gallium (Ga)-DOTATATE PET/CT or ${ }^{18}$ F-FDG (18-F-fluoro-deoxy-glucose) PET (100).

\section{Further considerations}

Several points need to be mentioned concerning this current complex topic since they are still a matter of discussions.

i) Tumors with neuroendocrine differentiation. Two special categories of malignancies have a distinct potential for neuroendocrine differentiation: malignant melanoma and breast cancer $(101,102)$. Because the fact that bone involvement, especially metastases, may be related to a neuroendocrine but also to a non-neuroendocrine component, we did not introduce them in the previous data (103). Under these circumstances, multiple additional mechanisms for bone loss are listed as the use of MEK inhibitors in melanoma, and prescription of aromatase inhibitors and induced hypogonadism in females of reproductive age with breast cancer $(104,105)$.

ii) NENs and primary hyperparathyroidism. Another cause of secondary osteoporosis in patients with NENs is related to the co-presence of PHP (106). This may be a well-known syndromic situation such as MEN type 1, an inherited autosomal dominant condition, also associating pancreatic NEN of different types and pituitary tumors, or MEN 2A, or it may be a less described association of NEN and another PTH-producing tumor (or even an incidental overlap) (107,108). Among MEN1-related hypophyseal neoplasia, the subjects with acromegaly, prolactinoma and Cushing disease are also candidates for secondary osteoporosis through multiple non-PTH-related mechanisms $(109,110)$.

iii) NENs and skeletal malformations. Another type of bone anomalies in NEN patients is represented by different malformations (111-113). The typical association of neurofibromatosis type 1 and NENs also involves scoliosis, long bone anomalies or other forms of skeleton dysplasia (111-113).

iv) Serotonin effects and bone metabolism. Serotonin (also known as 5-hydroxytriptamin, a tryptophan metabolite) is excessively produced by NENs manifested with carcinoid syndrome (especially GEP-NEN) which includes diarrhea, also causing nutritional impairment and weight loss that contribute to bone deterioration (114). However, serotonin also acts on bone, the gut-product has a direct action and it causes bone loss (every bone cell is equipped with at least one receptor for 5-hydroxytriptamin), while central (brain) serotonin (which actually does not directly communicate to circulating serotonin) has an indirect effect on bone increase (115). The kynurenin pathway, the other tryptophan metabolite, inhibits osteoblasts, also contributing to bone mass deterioration $(115,116)$. However, clinical studies on osteoporotic menopausal women did not reveal clear correlations with bone turnover markers, neither with DXA-BMD, because it seems that the skeleton influence is more complex than reflected by single circulating 5-hydroxytriptamine assays (116). Other medical circumstances involving anomalies of serotonin metabolism showed that, for instance, selective serotonin reuptake inhibitors (SSRIs), anti-depression drugs, are correlated with a higher fracture risk (117). But, currently we do not have enough studies to emphasis that excessive gut-derivate serotonin in NENs with carcinoid syndrome is an activator of bone loss thus a contributor to carcinoid-related osteoporosis (118). Whether this represents a pivotal element is still an open question.

\section{Conclusions}

Lately, due to a more complex and efficient therapeutic approach toward NENs, patient survival has improved; thus, there is a need for increasing the quality of patient life, including the bone status, but not only related to skeleton metastases, but also in those cases with a good prognosis related to carcinoid-related osteoporosis, knowing the burden of low-trauma fractures. Protocols of serial checking of fracture risk are imperious, especially if clinical risk factors that are more frequent in NEN patients are already identified (advanced age, diabetes mellitus, extended period of time in menopause, induced male/female hypogonadism, prolonged exposure to glucocorticoids). As simple tools of bone status assessment, biochemistry parameters such as serum calcium and bone turnover markers are extremely useful, in addition to other measurements such as DXA-BMD or even TBS (especially in menopausal diabetic women, PHEO/PG or ectopic Cushing syndrome). Up to this moment, carcinoid-related osteoporosis is not a standardized cause of secondary osteoporosis, but this remains an open topic and the future will provide us with specific data that require a multidisciplinary medical team once again, as usually NENs do.

\section{Acknowledgements}

Not applicable.

\section{Funding}

No funding was received.

\section{Availability of data and materials}

Not applicable.

\section{Authors' contributions}

AG drafted the manuscript and critically revised the final form. MC drafted the manuscript and revised the literature findings. FS revised the references and she serves as corresponding author. RCP researched the literature and organized the micro-chapters. AMO reviewed the literature findings and revised the final form of the manuscript. AV reviewed the 
literature findings and critically revised the final form of the manuscript and approved the final form. All authors read and approved the final manuscript for publication.

\section{Ethics approval and consent to participate}

Not applicable.

\section{Patient consent for publication}

Not applicable.

\section{Competing interests}

The authors declare that they have no competing interests.

\section{References}

1. Oberndorfer S: Karzinoide tumoren des dünndarms. In: Frankfurter Zeitschrift für Pathologie, pp426-429, 1907.

2. Gill AJ: Why did they change that? Practical implications of the evolving classification of neuroendocrine tumours of the gastrointestinal tract. Histopathology 78: 162-170, 2021.

3. Guadagno E, D'Avella E, Cappabianca P, Colao A and Del Basso De Caro M: Ki67 in endocrine neoplasms: To count or not to count, this is the question! A systematic review from the English language literature. J Endocrinol Invest 43: 1429-1445, 2020.

4. Assarzadegan $\mathrm{N}$ and Montgomery E: What is new in the 2019 World Health Organization (WHO) classification of tumors of the digestive system: Review of selected updates on neuroendocrine neoplasms, appendiceal tumors, and molecular testing. Arch Pathol Lab Med 145: 664-677, 2021.

5. Zappalà G, McDonald PG and Cole SW: Tumor dormancy and the neuroendocrine system: An undisclosed connection? Cancer Metastasis Rev 32: 189-200, 2013.

6. Ramírez-Rentería C, Ferreira-Hermosillo A,Marrero-Rodríguez D, Taniguchi-Ponciano K, Melgar-Manzanilla V and Mercado M: An update on gastroenteropancreatic neuroendocrine neoplasms: From mysteries to paradigm shifts. Arch Med Res 51: 765-776, 2020.

7. Klöppel $\mathrm{G}$ and La Rosa S: Ki67 labeling index: Assessment and prognostic role in gastroenteropancreatic neuroendocrine neoplasms. Virchows Arch 472: 341-349, 2018.

8. Kim JY, Hong SM and Ro JY: Recent updates on grading and classification of neuroendocrine tumors. Ann Diagn Pathol 29: 11-16, 2017.

9. Chauhan A, Yu Q, Ray N, Farooqui Z, Huang B, Durbin EB, Tucker T, Evers M, Arnold S and Anthony LB: Global burden of neuroendocrine tumors and changing incidence in Kentucky. Oncotarget 9: 19245-19254, 2018.

10. Sackstein PE, O'Neil DS, Neugut AI, Chabot J and Fojo T: Epidemiologic trends in neuroendocrine tumors: An examination of incidence rates and survival of specific patient subgroups over the past 20 years. Semin Oncol 45: 249-258, 2018.

11. Riihimäki M, Hemminki A, Sundquist K, Sundquist J and Hemminki K: The epidemiology of metastases in neuroendocrine tumors. Int J Cancer 139: 2679-2686, 2016.

12. Akimoto J, Fukuhara H, Suda T, Nagai K, Ichikawa M, Fukami S, Kohno M, Matsubayashi J and Nagao T: Clinicopathological analysis in patients with neuroendocrine tumors that metastasized to the brain. BMC Cancer 16: 36, 2016.

13. Cives M, Quaresmini D, Rizzo FM, Felici C, D'Oronzo S, Simone V and Silvestris F: Osteotropism of neuroendocrine tumors: Role of the CXCL12/ CXCR4 pathway in promoting EMT in vitro. Oncotarget 8: 22534-22549, 2017.

14. Cives M, Pellè E, Rinzivillo M, Prosperi D, Tucci M, Silvestris F and Panzuto F: Bone metastases in neuroendocrine tumors: Molecular parhogenesis and implications in clinical practice. Neuroendocrinology 111: 207-216, 2021.

15. Harvey NC, McCloskey EV, Mitchell PJ, Dawson-Hughes B, Pierroz DD, Reginster JY, Rizzoli R, Cooper C and Kanis JA: Mind the (treatment) gap: A global perspective on current and future strategies for prevention of fragility fractures. Osteoporos Int 28: $1507-1529,2017$
16. Liu J, Curtis EM, Cooper C and Harvey NC: State of the art in osteoporosis risk assessment and treatment. J Endocrinol Invest 42: 1149-1164, 2019.

17. Kanis JA, Svedbom A, Harvey N and McCloskey EV: The osteoporosis treatment gap. J Bone Miner Res 29: 1926-1928, 2014.

18. International Osteoporosis Foundation: https://www.osteoporosis.foundation/. Accessed April 1, 2021.

19. Clynes MA, Harvey NC, Curtis EM, Fuggle NR, Dennison EM and Cooper C: The epidemiology of osteoporosis. Br Med Bull 133: 105-117, 2020.

20. Curtis EM, Woolford S, Holmes C, Cooper C and Harvey NC: General and specific considerations as to why osteoporosis-related care is often suboptimal. Curr Osteoporos Rep 18: 38-46, 2020.

21. Barton DW, Behrend CJ and Carmouche JJ: Rates of osteoporosis screening and treatment following vertebral fracture. Spine J 19: 411-417, 2019.

22. Söreskog E, Borgström F, Shepstone L, Clarke S, Cooper C, Harvey I, Harvey NC, Howe A, Johansson H, Marshall T, et al: Long-term cost-effectiveness of screening for fracture risk in a UK primary care setting: the SCOOP study. Osteoporos Int 31: 1499-1506, 2020.

23. Zamborsky R, Svec A, Kokavec M and Galbavy S: Bone metastases in neuroendocrine tumors. Bratisl Lek Listy 118: 529-534, 2017.

24. Koizumi M, Gokita T and Toda K: Impending atypical femoral fracture in patients with medullary thyroid cancer with skeletal metastasis treated with long-term bisphosphonate and denosumab. Clin Nucl Med 42: 463-464, 2017.

25. Carsote M, Valea A, Dumitru N, Terzea D, Petrova E, Albu S, Buruiana A and Ghemigian A: Metastases in daily endocrine practice. Arch Balk Med Union 51: 476-480, 2016.

26. Rizzo FM, Vesely C, Childs A, Marafioti T, Khan MS, Mandair D, Cives M, Ensell L, Lowe H, Akarca AU, et al: Circulating tumour cells and their association with bone metastases in patients with neuroendocrine tumours. Br J Cancer 120: 294-300, 2019.

27. Zheng Z, Chen C, Jiang L, Zhou X, Dai X, Song Y and Li Y: Incidence and risk factors of gastrointestinal neuroendocrine neoplasm metastasis in liver, lung, bone, and brain: A population-based study. Cancer Med 8: 7288-7298, 2019.

28. Van Loon K, Zhang L, Keiser J, Carrasco C, Glass K, Ramirez MT, Bobiak S, Nakakura EK, Venook AP, Shah MH and Bergsland EK: Bone metastases and skeletal-related events from neuroendocrine tumors. Endocr Connect 4: 9-17, 2015.

29. Hori T, Yasuda T, Suzuki K, Kanamori M and Kimura T: Skeletal metastasis of carcinoid tumors: Two case reports and review of the literature. Oncol Lett 3: 1105-1108, 2012.

30. Trikalinos NA, Tan BR, Amin M, Liu J, Govindan R and Morgensztern D: Effect of metastatic site on survival in patients with neuroendocrine neoplasms (NENs). An analysis of SEER data from 2010 to 2014. BMC Endocr Disord 20: 44, 2020.

31. Wang S, Zhang J, Liu S and Zhang J: The prognostic analysis of different metastatic patterns in pancreatic neuroendocrine tumors patients: A population based analysis. Medicine (Baltimore) 98: e17773, 2019.

32. Deleval N, Pesque L, Dieudonné A, Viry F, Hentic O, Lebtahi R, Ruszniewski P and de Mestier L: Prognostic impact of bone metastases detected by ${ }^{18} \mathrm{~F}$-DOPA PET in patients with metastatic midgut neuroendocrine tumors. Eur Radiol 31: 4166-4174, 2021.

33. Altieri B, Di Dato C, Modica R, Bottiglieri F, Di Sarno A, Pittaway JFH, Martini C, Faggiano A and Colao A: Bone metabolism and vitamin D implication in gastroenteropancreatic neuroendocrine tumors. Nutrients 12: 1021, 2020.

34. Sen Gupta P, Grozinsky-Glasberg S, Drake WM, Akker SA, Perry L, Grossman AB and Druce MR: Are serotonin metabolite levels related to bone mineral density in patients with neuroendocrine tumours? Clin Endocrinol (Oxf) 80: 246-252, 2014.

35. Jacobson A and Cunningham JL: Connective tissue growth factor in tumor pathogenesis. Fibrogenesis Tissue Repair 5 (Suppl 1): S8, 2012.

36. Migut AE, Kaur H and Avritscher R: Neuroendocrine tumors: Imaging of treatment and follow-up. Radiol Clin North Am 58: 1161-1171, 2020.

37. Galgano SJ, Sharbidre K and Morgan DE: Multimodality imaging of neuroendocrine tumors. Radiol Clin North Am 58: 1147-1159, 2020.

38. Albu SE, Geleriu A, Carsote M, Mihai A, Vasiliu C and Poiana C: The vitamin D status in menopausal women. Arch Balk MedUnion 50: 275-277, 2015.

39. Khashayar P, Meybodi HA, Amoabediny G and Larijani B: Biochemical markers of bone turnover and their role in osteoporosis diagnosis: A narrative review. Recent Pat Endocr Metab Immune Drug Discov 9: 79-89, 2015. 
40. Centre for Metabolic Bone Diseases, University of Sheffield, UK: FRAX ${ }^{\circledR}$ Fracture Risk Assessment Tool: https://www.sheffield.ac.uk/FRAX/tool.aspx?country=9. Accessed April 1, 2021.

41. Carsote M, Radu O, Dumitrascu A, Terzea D, Valea A and Ghemigian A: Bone and menopause: Threshold of intervention. Rom Med J 63: 233-236, 2016.

42. Sandru F, Carsote M, Albu SE, Valea A, Petca A and Dumitrascu MC: Glucagonoma: From skin lesions to the neuroendocrine component (Review). Exp Ther Med 20: 3389-3393, 2020.

43. Byun J, Kim SH, Jeong HS, Rhee Y, Lee WJ and Kang CM: ACTH-producing neuroendocrine tumor of the pancreas: A case report and literature review. Ann Hepatobiliary Pancreat Surg 21: 61-65, 2017.

44. Sandru F, Carsote M, Dumitrascu MC, Albu SE and Valea A: Glucocorticoids and trabecular bone score. J Med Life 13: 449-453, 2020

45. Kannivelu A, Loke KS, Kok TY, Osmany SY, Ali SZ, Suat-Jin L and $\mathrm{Ng}$ DC: The role of PET/CT in the evaluation of skeletal metastases. Semin Musculoskelet Radiol 18: 149-165, 2014.

46. Adkins J and Lu Y: Diffuse bone metastases in pancreatic neuroendocrine tumor shown on octreoscan. Clin Nucl Med 44: 257-258, 2019.

47. Gauthé M, Testart Dardel N, Ruiz Santiago F, Ohnona J, Nataf V, Montravers F and Talbot JN: Vertebral metastases from neuroendocrine tumours: How to avoid false positives on ${ }^{68} \mathrm{Ga}$-DOTA-TOC PET using CT pattern analysis? Eur Radiol 28: 3943-3952, 2018

48. Lim KHJ, Raja H, D'Arienzo P, Barriuso J, McNamara MG, Hubner RA, Mansoor W, Valle JW and Lamarca A: Identification of areas for improvement in the management of bone metastases in patients with neuroendocrine neoplasms Neuroendocrinology 110: 688-696, 2020.

49. Altieri B, Di Dato C, Martini C, Sciammarella C, Di Sarno A Colao A and Faggiano A; NIKE Group: Bone metastases in neuroendocrine neoplasms: From pathogenesis to clinical management. Cancers (Basel) 11: 1332, 2019.

50. Alexandraki KI, Pizanias M, Uri I, Thomas D, Page T, Kolomodi D, Low CS, Adesanya O, Tsoli M, Gross DJ, et al: The prognosis and management of neuroendocrine neoplasms-related metastatic bone disease: Lessons from clinical practice. Endocrine 64: 690-701, 2019.

51. Zhang M, Zhao P, Shi X, Zhao A, Zhang L and Zhou L: Clinicopathological features and prognosis of gastroenteropancreatic neuroendocrine neoplasms in a Chinese population: A large, retrospective single-centre study. BMC Endocr Disord 17: $39,2017$.

52. Helbig G, Straczyńska-Niemiec A, Szewczyk I, Nowicka E Bierzyńska-Macyszyn G and Kyrcz-Krzemień S: Unexpected cause of anemia: Metastasis of neuroendocrine tumor to the bone marrow. Pol Arch Med Wewn 124: 635-636, 2014.

53. Valea A, Carsote M, Ghervan C and Georgescu C: Glycemic profile in patients with acromegaly treated with somatostatin analogue. J Med Life 8: 82-86, 2015.

54. Lamarca A, Elliott E, Barriuso J, Backen A, McNamara MG, Hubner R and Valle JW: Chemotherapy for advanced non-pancreatic well-differentiated neuroendocrine tumours of the gastrointestinal tract, a systematic review and meta-analys: A lost cause? Cancer Treat Rev 44: 26-41, 2016.

55. Poiană C, Carşote M, Ardeleanu C, Terzea D, Avramescu ET, Neamţu MC and Miulescu RD: The value of the immunohistochemistry in a case of gastric neuroendocrine tumor and thyroid metastasis. Rom J Morphol Embryol 52: 187-192, 2011

56. Watts NB: Postmenopausal osteoporosis: A clinical review. J Womens Health (Larchmt) 27: 1093-1096, 2018.

57. Misiorowski W: Osteoporosis in men. Prz Menopauzalny 16: 70-73, 2017.

58. Cortellini A, Bozzetti F, Palumbo P, Brocco D, Di Marino P, Tinari N, De Tursi M, Agostinelli V, Patruno L, Valdesi C, et al: Weighing the role of skeletal muscle mass and muscle density in cancer patients receiving PD-1/PD-L1 checkpoint inhibitors: A multicenter real-life study. Sci Rep 10: 1456, 2020.

59. Fernández-Sala X, Barceló-Vidal J, Tusquets $I$ and Conde-Estévez D: Effectiveness and safety of a novel dexamethasone mouthwash formulation in managing stomatitis in cancer patients. Farm Hosp 45: 41-44, 2020.

60. Conteduca V, Oromendia C, Eng KW, Bareja R, Sigouros M, Molina A, Faltas BM, Sboner A, Mosquera JM,Elemento O, et al: Clinical features of neuroendocrine prostate cancer. Eur J Cancer 121: 7-18, 2019.
61. Clement DS, Tesselaar ME, van Leerdam ME, Srirajaskanthan R and Ramage JK: Nutritional and vitamin status in patients with neuroendocrine neoplasms. World J Gastroenterol 25: 1171-1184, 2019.

62. Kędzierska L,Madej-Michniewicz A, Marczuk N, Dołęgowska B, Starzyńska T and Błogowski W: Clinical significance of various growth factors in patients with different gastric neoplasms. Am J Transl Res 12: 118-129, 2020.

63. Ranade R and Basu S: Metabolic bone disease in the context of metastatic neuroendocrine tumor: Differentiation from skeletal metastasis, the molecular PET-CT imaging features, and exploring the possible etiopathologies including parathyroid adenoma (MEN1) and paraneoplastic humoral hypercalcemia of malignancy due to PTHrP hypersecretion. World J Nucl Med 16: $62-67,2017$

64. Iliesiu A, Ungureanu IA, Petca A, Constantin MM, Petca RC, Sandru F, Constantin T and Dumitrascu MC: Paraganglioma presenting as a mesenteric cystic mass: A case report. Exp Ther Med 20: 2489-2492, 2020.

65. Valea A, Morar A, Ghemigian A, Petrova E, Tupea C, Popescu M, Dumitru N and Carsote M: Diagnosis of neurofibromatosis type 1-related pheocromocytoma after stroke and myocardical infarction. Rom Med J 67: 420-424, 2020.

66. Aygun $\mathrm{N}$ and Uludag M: Pheochromocytoma and paraganglioma: From epidemiology to clinical findings. Sisli Etfal Hastan Tip Bul 54: 159-168, 2020.

67. Sandru F, Dumitrascu MC, Albu SE, Carsote M and Valea A: Osteoporosis and adrenal incidentaloma: To be or not to be? Rom J Med Pract 14: 381-384, 2019.

68. Jimenez P, Tatsui C, Jessop A, Thosani S and Jimenez C: Treatment for malignant pheochromocytomas and paragangliomas: 5 Years of progress. Curr Oncol Rep 19: 83, 2017.

69. Ilanchezhian M, Jha A, Pacak K and Del Rivero J. Emerging treatments for advanced/metastatic pheochromocytoma and paraganglioma. Curr Treat Options Oncol 21: 85, 2020.

70. Rider AJ, Walsh A, Sollenberger EL, Dryden SC, DeAngelis KD, Weir AB III and Fowler BT: Orbital pheochromocytoma metastasis in 2 patients with known pheochromocytoma. Ophthalmic Plast Reconstr Surg 35: e131-e134, 2019.

71. Rahpeyma A and Khajehahmadi S: Pheochromocytoma metastasing to the jaw: Review of the literature and new case. Indian J Surg Oncol 11: 433-437, 2020.

72. Fadiga L, Saraiva J, Paiva I and Carrilho F: Thoracic spine metastasis presenting 18 years after complete resection of a phaeochromocytoma. BMJ Case Rep 12: e229621, 2019.

73. Jang Khan NA, Ullah S, Siddiqui HU and Karim A: Spinal cord compression by metastatic thoracic spine paraganglioma. J Ayub Med Coll Abbottabad 28: 617-619, 2016.

74. Ayala-Ramirez M, Palmer JL, Hofmann MC, de la Cruz M, Moon BS, Waguespack SG, Habra MA and Jimenez C: Bone metastases and skeletal-related events in patients with malignant pheochromocytoma and sympathetic paraganglioma. J Clin Endocrinol Metab 98: 1492-1497, 2013.

75. Huang CT, Yang WC and Lin SF: Multiple endocrine neoplasia type 2A. Kaohsiung J Med Sci 28: 341-344, 2012.

76. Gravel G, Leboulleux S, Tselikas L, Fassio F, Berraf M, Berdelou A, Ba B, Hescot S, Hadoux J, Schlumberger M, et al: Prevention of serious skeletal-related events by interventional radiology techniques in patients with malignant paraganglioma and pheochromocytoma. Endocrine 59: 547-554, 2018.

77. Athimulam S and Bancos I: Evaluation of bone health in patients with adrenal tumors. Curr Opin Endocrinol Diabetes Obes 26: 125-132, 2019.

78. Kim BJ, Kwak MK, Kim JS, Lee SH and Koh JM: Higher sympathetic activity as a risk factor for skeletal deterioration in pheochromocytoma. Bone 116: 1-7, 2018.

79. Yokomoto-Umakoshi M, Umakoshi H, Fukumoto T, Matsuda Y, Nagata H, Ogata M, Kawate H, Miyazawa T, Sakamoto R and Ogawa Y; Q-AND-A study group: Pheochromocytoma and paraganglioma: An emerging cause of secondary osteoporosis. Bone 133: 115221, 2020.

80. Yokomoto-Umakoshi M,Umakoshi H, Sakamoto R, Fukumoto T, Ogata M, Nakano Y, Iwahashi N, Kaneko H, Mizoguchi N, Hattori A, et al: Role of deteriorated bone quality in the development of osteoporosis in pheochromocytoma and paraganglioma. Bone 142: 115607, 2021

81. Yokomoto-Umakoshi M, Umakoshi H, Ogata M, Fukumoto T, Matsuda Y, Miyazawa T, Sakamoto R and Ogawa Y; Q-AND-A study group: Coexistence of osteoporosis and atherosclerosis in pheochromocytoma: New insights into its long-term management. Osteoporos Int 31: 2151-2160, 2020. 
82.Poiana C, Radoi V, Carsote $\mathrm{M}$ and Bilezikian JP: New clues that may link osteoporosis to the circulating lipid profile. Bone Res 1: 260-266, 2013.

83. Jackuliak P, Kužma M, Killinger Z and Payer J: Good long-term glycemic compensation is associated with better trabecular bone score in postmenopausal women with type 2 diabetes. Physiol Res 68 (Suppl 2): S149-S156, 2019.

84. Webb SM and Valassi E: Morbidity of Cushing's syndrome and impact of treatment. Endocrinol Metab Clin North Am 47: 299-311, 2018.

85. Dumitrascu MC, Stanescu AMA, Bejan C, Sandru F, Toader DO, Radavoi DG, Cotirlet A, Judea Pusta CT and Diaconu CC: Obesity and its implications on stress urinary incontinence. Rev Chim 70: 3660-3662, 2019.

86. Young J, Haissaguerre M, Viera-Pinto O, Chabre O, Baudin E and Tabarin A: Management of endocrine disease: Cushing's syndrome due to ectopic ACTH secretion: An expert operational opinion. Eur J Endocrinol 182: R29-R58, 2020.

87. Grigoryan S, Avram AM and Turcu AF: Functional imaging in ectopic Cushing syndrome. Curr Opin Endocrinol Diabetes Obes 27: 146-154, 2020.

88. Kyriacou A, Stepien KM and Issa B: Urinary steroid metabolites in a case of florid ectopic Cushing's syndrome and clinical correlations. Hormones (Athens) 15: 540-547, 2016.

89. Hayes AR and Grossman AB: The ectopic adrenocorticotropic hormone syndrome: Rarely easy, always challenging. Endocrinol Metab Clin North Am 47: 409-425, 2018.

90. Sandru F, Dumitrascu MC, Albu SE, Carsote M and Valea A: Obesity and osteoporotic fractures. Rom J Mil Med 123 166-171, 2020.

91. Carsote M, Preda SA, Mitroi M, Camen A and Radu L: Serum osteocalcin, P1NP, alkaline phosphase, and crosslaps in humans: The relationship with body mass index. Rev Chim 70 1615-1618, 2019.

92. Broersen LHA, van Haalen FM, Kienitz T, Biermasz NR, Strasburger CJ, Dekkers OM and Pereira AM: Sex Differences in presentation but not in outcome for ACTH-dependent Cushing's syndrome. Front Endocrinol (Lausanne) 10: 580, 2019.

93. Vinolas H, Grouthier V, Mehsen-Cetre N, Boisson A Winzenrieth R, Schaeverbeke T, Mesguich C, Bordenave L and Tabarin A: Assessment of vertebral microarchitecture in overt and mild Cushing's syndrome using trabecular bone score. Clin Endocrinol (Oxf) 89: 148-154, 2018.

94. Valassi E, Santos A, Yaneva M, Tóth M, Strasburger CJ, Chanson P, Wass JA, Chabre O, Pfeifer M, Feelders RA, et al: The European registry on Cushing's syndrome: 2-Year experience. Baseline demographic and clinical characteristics. Eur J Endocrinol 165: 383-392, 2011.

95. Rashid F, Riccio SA, Munk PL, Malfair D and Heran MK Vertebroplasty for vertebral compression fractures secondary to Cushing's syndrome induced by an ACTH-producing bronchial carcinoid tumour. Singapore Med J 50: e147-e150, 2009.

96. Coe SG, Tan WW and Fox TP: Cushing's syndrome due to ectopic adrenocorticotropic hormone production secondary to hepatic carcinoid: Diagnosis, treatment, and improved quality of life. J Gen Intern Med 23: 875-878, 2008.

97. Neary NM, Lopez-Chavez A, Abel BS, Boyce AM, Schaub N, Kwong K, Stratakis CA, Moran CA, Giaccone G and Nieman LK: Neuroendocrine ACTH-producing tumor of the thymus-experience with 12 patients over 25 years. J Clin Endocrinol Metab 97: 2223-2230, 2012.

98. Gut P: Liver metastases in gastroenteropancreatic neuroendocrine tumours-treatment methods. Prz Gastroenterol 15 207-214, 2020.

99. Carsote M, Petrova EN, Radu O, Goldstein A, Ghemigian A and Valea A: Serotonin: Beyond menopause: Rom J Med Pract (Pract Med) 11: 270-272, 2016.

100. Szajer J, Yung G, Russo R and Mansberg R: Adrenocorticotropic hormone-secreting neuroendocrine tumor of the rectum demonstrated on 68Ga-DOTATATE and 18F-FDG PET imaging. Clin Nucl Med 42: 773-775, 2017.
101. Angarita FA, Rodríguez JL, Meek E, Sánchez JO, Tawil M and Torregrosa L: Locally-advanced primary neuroendocrine carcinoma of the breast: Case report and review of the literature. World J Surg Oncol 11: 128, 2013.

102. Banerjee SS and Eyden B: Divergent differentiation in malig nant melanomas: A review. Histopathology 52: 119-129, 2008.

103. Poiană C, Neamţu MC, Avramescu ET, Carşote M, Trifănescu R, Terzea D, Neamtu OM and Dănciulescu Miulescu R: The dedifferentiation of neuroendocrine tumor metastases: Myth or reality? Rom J Morphol Embryol 54: 201-203, 2013.

104. Dumas M, Laly P, Gottlieb J, Vercellino L, Paycha F, Bagot M, Baroudjian B, Madelaine I, Basset-Seguin N, Eftekhari P, et al: Osteopenia and fractures associated with long-term therapy with MEK inhibitors. Melanoma Res 28: 641-644, 2018.

105. Lee YK, Lee EG, Kim HY, Lee Y, Lee SM, Suh DC, Yoo JI and Lee S: Osteoporotic fractures of the spine, hip, and other locations after adjuvant endocrine therapy with aromatase inhibitors in breast cancer patients: A meta-analysis. J Korean Med Sci 35: e403, 2020.

106. Li Y and Simonds WF: Endocrine neoplasms in familial syndromes of hyperparathyroidism. Endocr Relat Cancer 23: R229-R247, 2016.

107. Miulescu R, Balaban DV, Sandru F and Jinga M: Cutaneous manifestations in pancreatic diseases-A review. J Clin Med 9: $2611,2020$.

108. Slouma M, Abbes M, Dhahri R, Litaiem N, Gueddiche N, Mansouri N, Msekni I, Gharsallah I, Metoui L and Louzir B: Multiple endocrine neoplasia type 1 revealed by a hip pathologic fracture. Clin Rheumatol 40: 775-782, 2021

109. Herath M, Parameswaran V, Thompson M, Williams M and Burgess J: Paediatric and young adult manifestations and outcomes of multiple endocrine neoplasia type 1 . Clin Endocrinol (Oxf) 91: 633-638, 2019.

110. Mele C, Mencarelli M, Caputo M, Mai S, Pagano L, Aimaretti G, Scacchi M, Falchetti A and Marzullo P: Phenotypes associated with MEN1 syndrome: A focus on genotype-phenotype correlations. Front Endocrinol (Lausanne) 11: 591501, 2020.

111. Sandru F, Carsote M, Valea A, Albu SE, Petca RC and Dumitrascu MC: Somatostatinoma: Beyond neurofibromatosis type 1 (review). Exp Ther Med 20: 3383-3388, 2020.

112. Shah $\mathrm{S}$ and George KJ: The association of spinal deformity with dural ectasia in neurofibromatosis type 1 . Br J Neurosurgery 33 : 620-623, 2019.

113. Prudhomme L, Delleci C, Trimouille A, Chateil JF, Prodhomme O, Goizet C and Van Gils J: Severe thoracic and spinal bone abnormalities in neurofibromatosis type 1 . Eur J Med Genet 63: 103815, 2020.

114. Eads JR, Reidy-Lagunes D, Soares HP, Chan JA, Anthony LB, Halfdanarson TR, Naraev BG, Wolin EM, Halperin DM, Li D, et al: Differential diagnosis of diarrhea in patients with neuroendocrine tumors. Pancreas 49: 1123-1130, 2020.

115. Michalowska M,Znorko B, Kaminski T, Oksztulska-Kolanek E and Pawlak D: New insights into tryptophan and its metabolites in the regulation of bone metabolism. J Physiol Pharmacol 66 779-791, 2015.

116. Al Saedi A, Sharma S, Summers MA, Nurgali K and Duque G: The multiple faces of tryptophan in bone biology. Exp Gerontol 129: 110778, 2020

117. Khanassov V, Hu J, Reeves D and van Marwijk H: Selective serotonin reuptake inhibitor and selective serotonin and norepinephrine reuptake inhibitor use and risk of fractures in adults: A systematic review and meta-analysis. Int J Geriatr Psychiatry 33: 1688-1708, 2018.

118. Banskota S, Ghia JE and Khan WI: Serotonin in the gut: Blessing or a curse. Biochimie 161: 56-64, 2019. 